\title{
Zebra Battery Technologies for the All Electric Smart Car
}

\author{
T. M. O’Sullivan, C. M. Bingham and R. E. Clark \\ Electrical Machines and Drives Group, Department of Electronic and Electrical Engineering, University of Sheffield, \\ Sheffield, S1 3JD, (UK)
}

\begin{abstract}
This paper describes the operational behaviour and advantages of the high temperature, Sodium Nickel Chloride (Zebra) battery, for use in all electric urban (city) vehicles. It is shown that an equivalent parallel electrical circuit can be employed to accurately simulate the electrochemical behaviour inherent in the most recent generation of Zebra cells. The experimental procedure is outlined and summary attributes of the investigation validated by both simulation studies, and experimentally, via measurements from a prototype battery module intended for use in an all electric smart car.
\end{abstract}

Index Terms-- Batteries, Electric propulsion, Energy storage, Equivalent circuits, Modelling.

\section{INTRODUCTION}

Due to increases in air pollution in congested cities, as well as decreases in fuel efficiency for car usage in these areas (i.e. traffic jams, stop lights, average speed, etc.) the potential of small urban cars with alternative, low carbon emission energy sources and drive train topologies have been heavily investigated in recent years.

The urban vehicle under consideration here is shown in Fig. 1, viz. all-electric (EV) prototype version of the smart car (a brand of Daimler Chrysler). The drive train, Fig. 2, designed by Zytek Electric Vehicles Ltd., is driven by a $55 \mathrm{~kW}(75 \mathrm{Hp}$ peak) permanent magnet brushless DC machine (BLDC) and supplied from an IGBT power inverter via a $26 \mathrm{~kW}(36 \mathrm{Hp})$ Zebra battery module, providing a DC bus voltage of $\approx 300 \mathrm{~V}$.

The progression of the EV and the hybrid electric vehicles (HEV) from prototype to commercial availability has been limited primarily by the deficiencies in current alternative energy storage technologies. These include high cost, limited life span and low energy density when compared with the internal combustion (IC) engine and carbon based fuel. Moreover, in recent years, with the availability of high-power silicon devices and advances in electrical machine design, such limitations have become more critical in hindering the successful development of the alternative energy approach, particularly for the EV. As a result, therefore, a compromise, i.e. the HEV (two energy sources, one of which is often a battery) solution has been the mainstream. However, such solutions, particularly for the urban vehicle market are usually complex, expensive, and

The authors acknowledge the financial support of the UK Department of Trade and Industry (DTI) and contributions of the project partners, Zytek Electric Vehicles Ltd., Beta Research and Development Ltd. and MIRA. for most driving cycles do not improve fuel economy or reduce emissions significantly over a small, welldesigned IC engine.

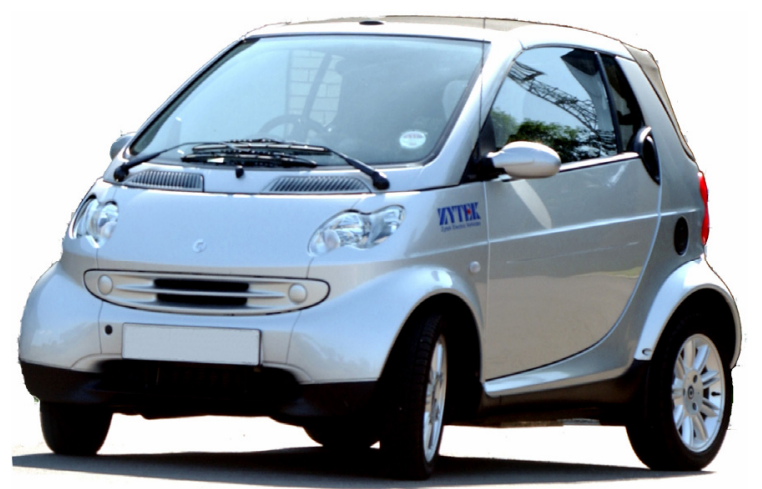

Fig. 1. All-electric smart car

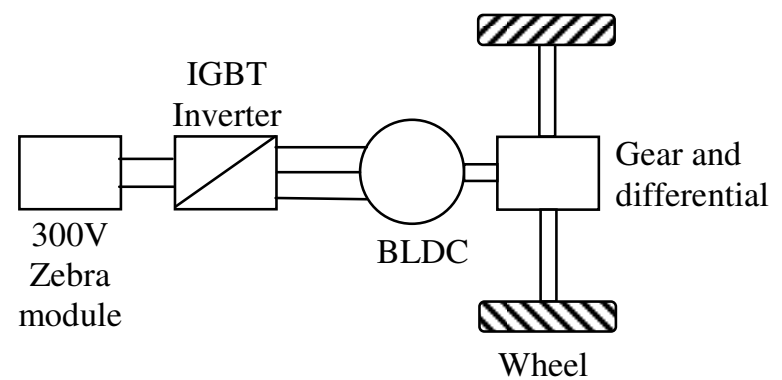

Fig. 2. Drive-train topology

The Zebra battery module, Fig. 3, has been previously utilised successfully in prototype EVs, such as the BMW E1 [1] and Mercedes-Benz 'A'-class as well as electric/hybrid public transportation buses in California and Italy. Additionally, Rolls Royce has selected the technology to replace lead-acid in military and rescue surface and submersible ship applications. It therefore promises to be a viable competitive energy storage solution for EV applications, with several added advantages over other battery technologies. For instance, a typical Zebra module [2,3], exhibits a high energy density of $120 \mathrm{Wh} / \mathrm{kg} \mathrm{3-4} \mathrm{times} \mathrm{higher} \mathrm{than} \mathrm{lead-acid} \mathrm{and}$ 2-3 times nickel-metal hydride [4]. The pulse power capability is also adequate for typical electric vehicles acceleration profiles, at around 1.5 the rated energy $(170 \mathrm{~W} / \mathrm{kg})$. Zebra cells also offer significantly increased cycle life $\approx 3500$ nameplate cycles (7-8 times higher than lead-acid), maintenance free operation, unaffected by ambient temperatures, no gassing, zero self-discharge and 
cells fail to a low resistance, so series connected strings containing failed cells will continue to operate. Of particular importance to EV applications, state of charge estimation is also simple to evaluate since the cell are $100 \%$ coulombically efficient i.e. Ah charge in $=\mathrm{Ah}$ discharge out.

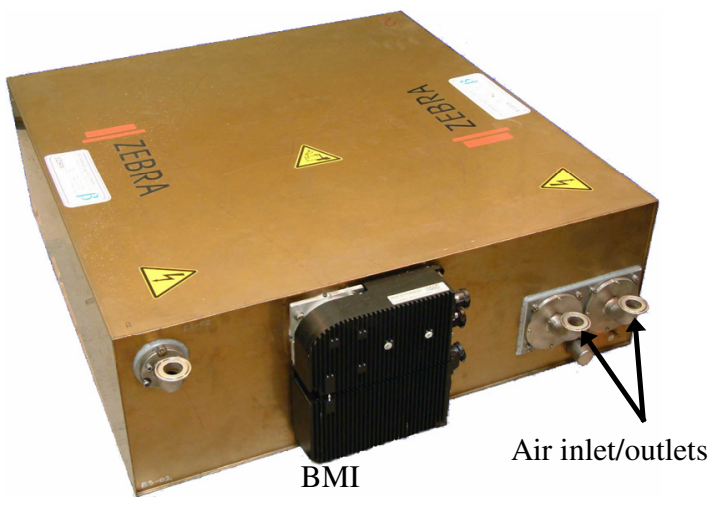

Fig. 3. Typical Zebra module

Individual Zebra cells operate within a specified temperature range of $270-350^{\circ} \mathrm{C}$ (as discussed in the next section). Series and parallel chains are interconnected and packaged within a sealed, vacuum insulated, air-cooled modular casing, Fig. 3, to minimise heat loss to the environment. The module is regulated by a dedicated battery management unit (BMI) attached to the front. The BMI is fully automated, controlling the charging, maximum and minimum terminal voltages during loading, charge status and thermal management by regulating internal heaters and fan cooling. However, in general use, once operating temperatures are reached, the internal temperature of the module is self-regulating, exhibiting a natural cooling of around $-3^{\circ} \mathrm{C} /$ hour (if left unconnected to an external power supply). Additionally, the module can remain within specified temperature limits during a one hour discharge rate without additional forced air cooling. Furthermore, after a sustained drive cycle, heat can be absorbed during the charging period (i.e. heat can be either evolved or absorbed during charging).

The relatively simple smart EV drive train, Fig. 2, has therefore become an increasingly viable solution when combined with a Zebra module, being particularly well suited for around $80 \%$ of all urban vehicle trips, i.e. shortdistance, high-use applications within 50-100 mile ranges. However, due to the complex nature, and recent development of the Zebra cell, there have been few equivalent models to predict its performance in applications such as EVs. To date therefore, much EV prototyping has been on a trial and error basis with time consuming experimental evaluation prohibiting an optimal solution and slowing progression. Furthermore, there have been limited publications that clearly demonstrate the potential of this relatively new technology.

\section{ZEBRA BATTERY EQUIVALENT CIRCUIT}

The purpose of this section is to explore the main electrochemical behaviour within the Zebra cell and formulate an equivalent electrical circuit representation.

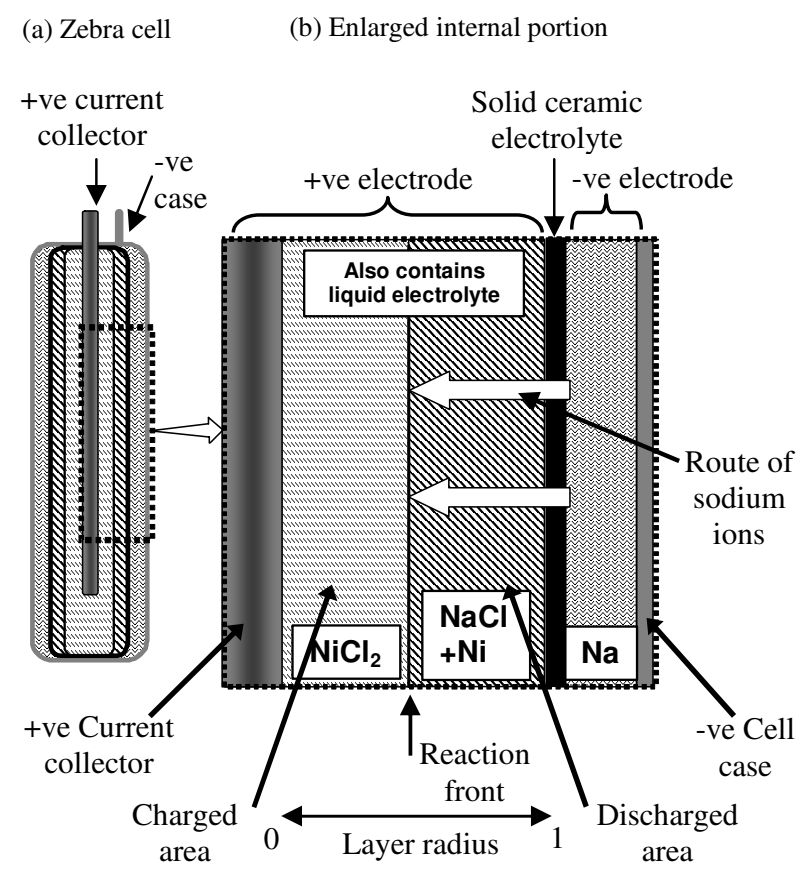

Fig. 4. Simplified chemical reactions within the Zebra cell during discharging

Figure 4 illustrates the Zebra cell showing (a) the main components of the cell and (b) an enlarged portion. The central solid positive (+ve) electrode consists of a nickel conductor (current collector) surrounded by a mixture of nickel chloride $\left(\mathrm{NiCl}_{2}\right)$, nickel $(\mathrm{Ni})$ and liquid electrolyte (enabling the transfer of $\mathrm{Na}^{+}$within the +ve electrode). The negative (-ve) electrode constitutes the metal cell casing and molten sodium $(\mathrm{Na})$. Both electrodes are separated via a solid ceramic electrolyte which facilitates the conduction of $\mathrm{Na}^{+}$ions between the reaction partners, sodium and nickel chloride. It also has the property of simultaneously acting as an insulator for electrons. The cell requires high temperatures to liquefy the sodium and ensure the internal resistance is minimised. The maximum temperature is dictated by the maximum allowable level of internal cell pressure.

During discharging, as illustrated by the events in Fig. 4, sodium reacts with nickel chloride to form sodium chloride (salt) and nickel. This process is reversed during charging and is given by (1).

$$
2 \mathrm{Na}+\mathrm{NiCl}_{2} \underset{\text { Charging }}{\stackrel{\text { Discharge }}{\longleftarrow}} 2 \mathrm{NaCl}+\mathrm{Ni}
$$

Variation of internal resistance is mainly attributed to the behaviour of the solid +ve electrode (a characteristic feature of Zebra cells). During the course of discharging, the reaction front migrates inwards towards the central current collector, as illustrated in Fig. 4. This migration 
increases the overall internal resistance of the +ve electrode since the reaction front is reduced, and the propagation distance of the $\mathrm{Na}+$ ions is increased. It can be seen therefore that the +ve electrode of a fully charged cell contains only $\mathrm{NiCl}_{2}$, whilst a fully discharged cell contains only $\mathrm{NaCl}$ and $\mathrm{Ni}$. The concept of reaction front is normalised and termed layer radius $\mathrm{Sr}$. When $\mathrm{Sr}=0$ the reaction front is directly adjacent to the central current collector and when $\mathrm{Sr}=1$ adjacent to the sold ceramic electrolyte.

During charging, a second charged area $\left(\mathrm{NiCl}_{2}\right.$ layer) is formed immediately adjacent to the solid ceramic electrolyte, irrespective of the depth of discharge (DOD) or the radius of the previous reaction front. Hence, after a short charge, the layer radius is identical to that of a fully charged cell. It follows therefore, that during a subsequent discharge, the cell exhibits a low internal resistance as it depletes the recently charged outermost layer. Once depleted, the reaction front (or layer radius) returns to the previous position before the charge was initiated. Such an event subsequently causes non-linear step terminal voltages during a driving cycle, creating the accumulation of many layers. As a result, the Zebra cell cannot be accurately described as a function of charge state, as with other cell types.

The above electrochemical processes can be simplified and represented as an equivalent electrical circuit of a cell as illustrated in Fig. 5. $V_{\text {term }}$ and $I_{\text {term }}$ are the terminal voltage and current, respectively, and $U_{N i}$ is the open circuit voltage (OCV) representing the electrochemical potential of sodium and nickel chloride. The passive electrical components consist of $R_{N i}$, the lumped ohmic resistance of the solid ceramic electrolyte and interconnecting metal, $R_{N i t}$, the resistance of the +ve electrode, and $\mathrm{C}_{\mathrm{Nit}}$, a capacitance which simulates the diffusion processes within the +ve electrode. However, this circuit which is termed the nickel cell cannot adequately represent the newest generation of Zebra cells which also contain iron doping of the positive electrode. The doping forms a second sodium/iron cell $\left(\mathrm{Na} / \mathrm{FeCl}_{2}\right)$ that takes part in the reaction directly adjacent to the solid ceramic electrolyte and constitutes approximately $20 \%$ of the total energy capacity in the most recent cells.

To simulate the parallel supposition of the two individual cells, the circuit of Fig. 5 is expanded in Fig. 6 where $R_{F e}$ and $U_{F e}$, represent the lumped iron cell resistance and OCV respectively. The iron cell only contributes to $I_{\text {term }}$ during a high power pulse discharge when the $V_{\text {term }}$ falls below $U_{F e}$, thereby enhancing the pulse power capability (when compared with an undoped cell). Immediately commencing a high power pulse discharge when $V_{\text {term }}$ increases above $U_{F e}$, the iron cell is recharged by via the nickel cell. Current flow during this event is illustrated by the dotted line in Fig. 6.

The level of iron doping is carefully prescribed, representing a trade-off based on performance and cost. Iron is cheaper than nickel and improves pulse power capability. However, an increase in iron reduces the capacity of the higher voltage nickel cell thereby influencing nameplate performance towards the end of DOD, when the capacity of the nickel cell has depleted.

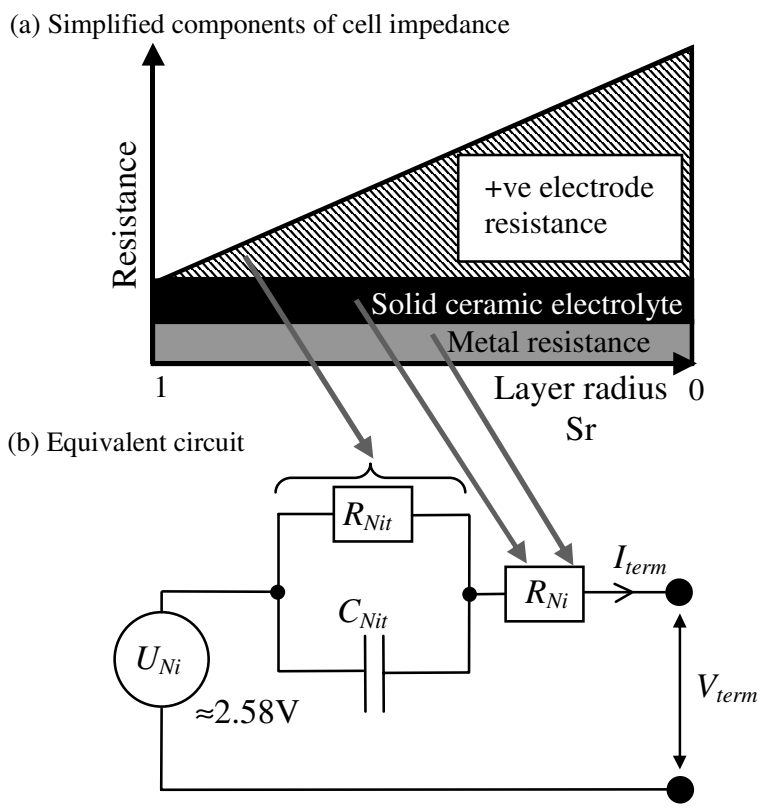

Fig. 5. Electrical circuit representation of a Zebra cell

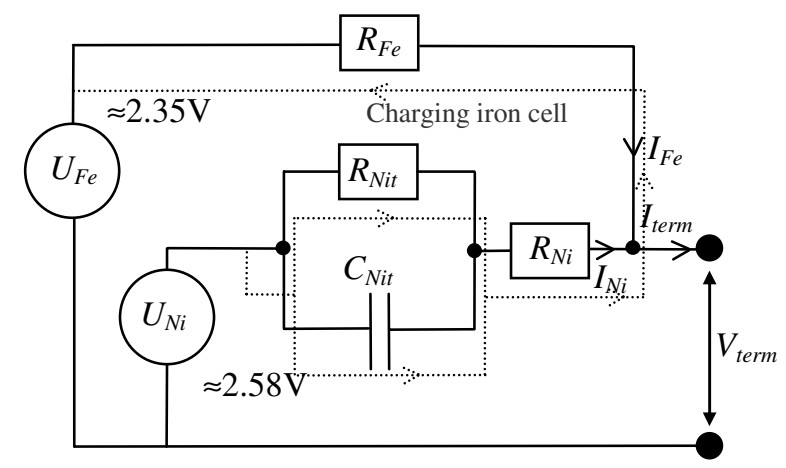

Fig. 6. Electric circuit representation of an iron doped Zebra cell

In general, both the liquid and ceramic electrolyte exhibit increased ion conductivity with elevated cell temperatures which has the effect of reducing impedance of the +ve electrode. Furthermore the OCV is also influenced by temperature variation. The dependences of the individual elements of the equivalent electrical circuit on additional parameters are assumed as follows [5]:

- OCV has a dependence on charge state (DOD) and temperature only.

- $\quad C_{N i t}, R_{N i t}$, and $R_{N i}$ are not dependant on DOD but show dependency on the current layer radius and temperature. Furthermore they exhibit different values for charging and discharging.

- $R_{F e}$, has shown dependency on current layer radius and the DOD of the iron cell (DOD_Fe). Temperature dependence is small and ignored. 


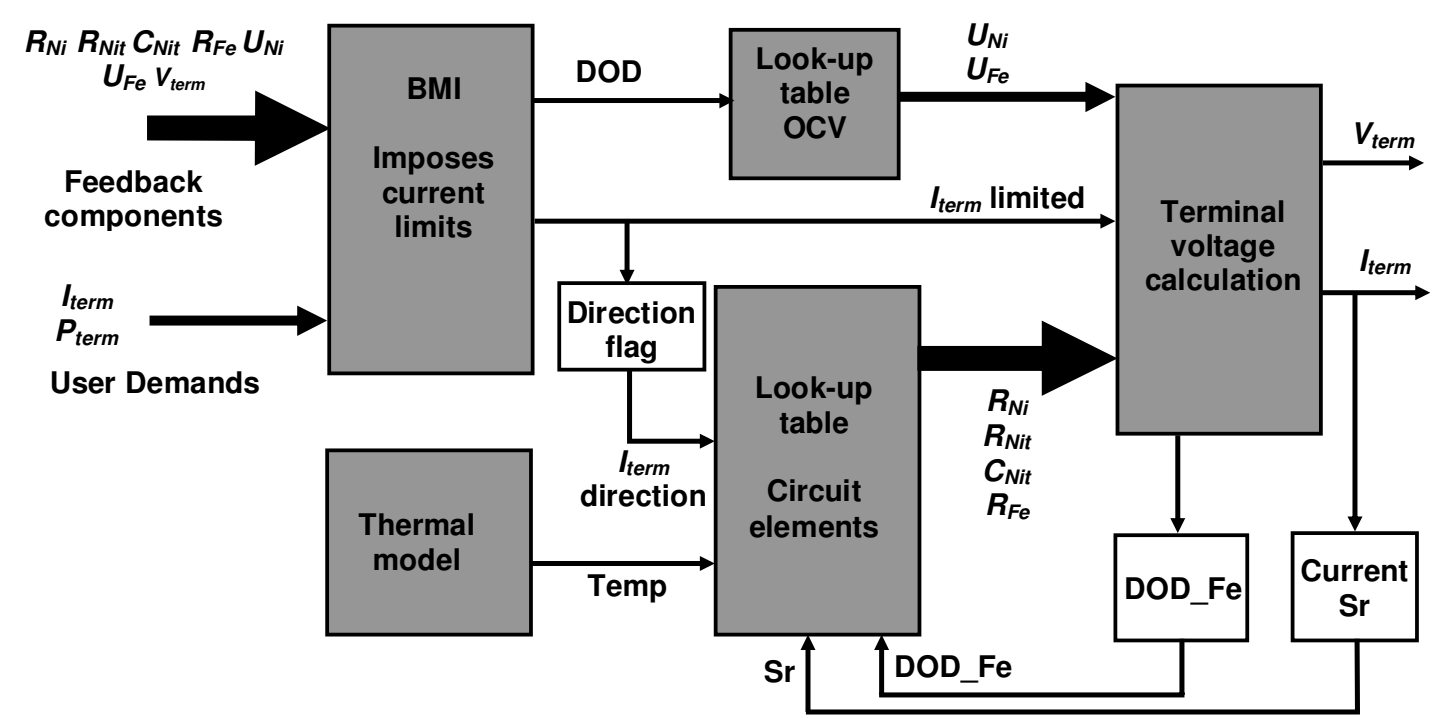

Fig. 7. Schematic of the Zebra battery model structure.

\section{ZEBRA BATTERY MODEL}

The model is based entirely on a single cell representation, which is scaled to simulate the interconnection of series and parallel chains. Figure 7 illustrates schematically the model structure which is implemented in a Simulink ${ }^{\circledR}$ modelling environment. The equivalent electric circuit model, Fig. 6, forms the core and is evaluated for each simulation step as a conventional linear (time invariant) circuit. However, for each step, the equivalent circuit parameters are modified based on the assumptions outlined in the previous section in accordance with variations in Sr, DOD, terminal current direction and battery temperature, obtained via an auxiliary thermal model. Of particular importance, the prior history, location and capacity (in Ah) of previously charged layers are stored. Outermost, charged layers are depleted first by initiating step changes in the current layer radius. The BMI is modelled based on the actual unit, enforcing voltage/current limits and monitoring state of charge etc. Model accuracy is determined by the estimated equivalent circuit parameters with respect to a given operating condition. Parameters are identified via terminal voltage data at discrete points and the model performs interpolation between adjacent values.

Experiments where preformed on a 10 cell series chain module, containing the most recent ML/8P 25Ah cells, (intended for use in future EV smart cars). The module has a nominal OCV of $25.8 \mathrm{~V}$. Figure 8 shows terminal voltage progression when the module is subjected to a repeating pulse discharge sequence of $60 \mathrm{~min}$ at $2.5 \mathrm{~A}$, $30 \mathrm{sec}$ at $12.5 \mathrm{~A}$ followed by $30 \mathrm{~min}$ open circuit. Notably these results where performed at a fixed temperature $\left(295^{\circ} \mathrm{C}\right)$ and the module was previously fully charged (i.e. contains no previous charged layers) and completely discharged within the loading sequence.

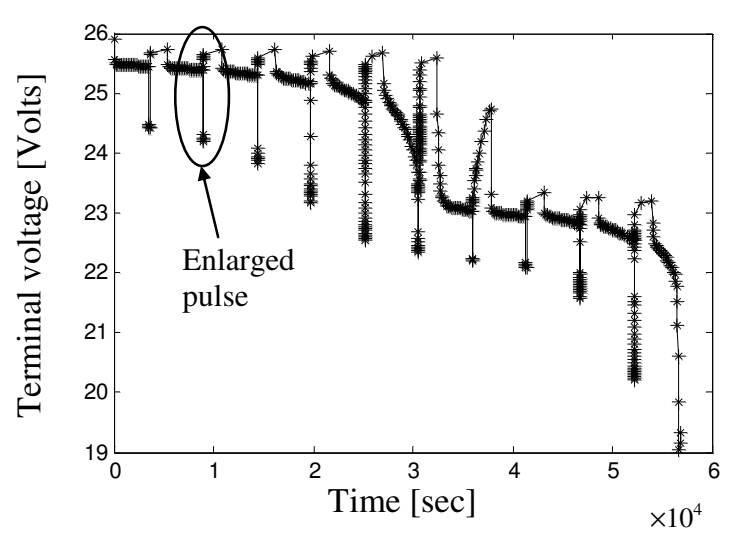

Fig. 8. Terminal voltage progression during a pulse discharge sequence

From the data in Fig. 8 the OCV as a function of DOD can be obtained as well as equivalent circuit parameters for the nickel cell as a function of layer radius. Notably, similar experiments were repeated at different temperatures employing both charging and discharging load sequences. One of the voltage transients during a 12.5A pulse in Fig. 8 (marked) is enlarged and shown in Fig. 9 alongside the method for calculating the nickel cell resistances. The capacitance is determined from the time constant $\tau$, by curve matching the transient part of the pulse.

In order to ensure the iron cell is not contributing to the terminal current (resulting in error of the estimated nickel cell resistances) the terminal voltage must be kept above $\approx 23.5 \mathrm{~V}$. Unfortunately voltages below $23.5 \mathrm{~V}$ are an inevitable occurrence at high DOD, since the nickel cell capacity is depleted, and the terminal current consists only of the iron cell contribution. This is characterised in 
Fig. 8 , by a sudden fall in OCV to $\approx 23.5 \mathrm{~V}$ (during the 30 min open circuit periods).

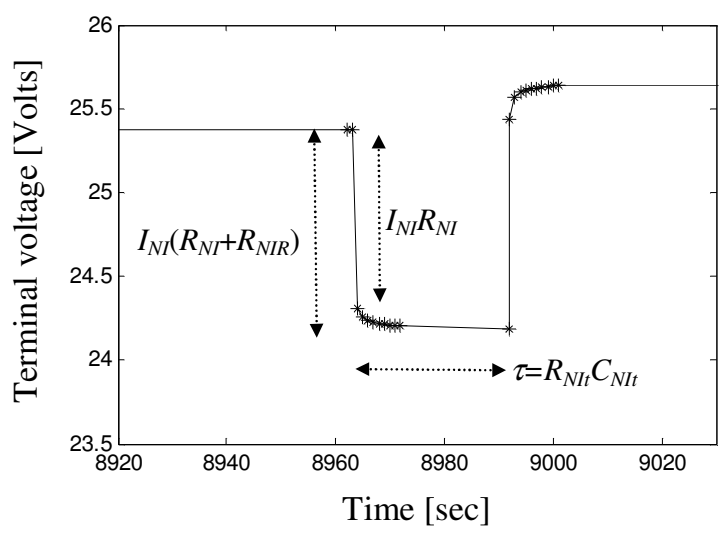

Fig. 9. Enlarged transient terminal voltage pulse

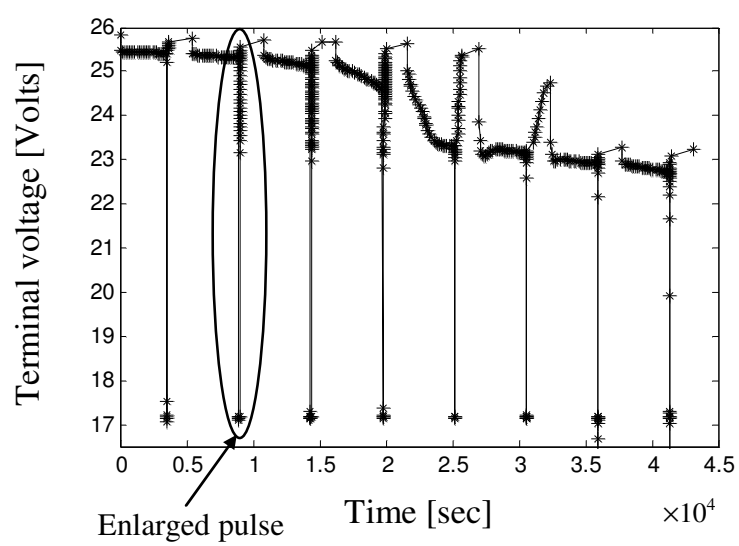

Fig. 10. Terminal voltage progression during a high power pulse discharge sequence

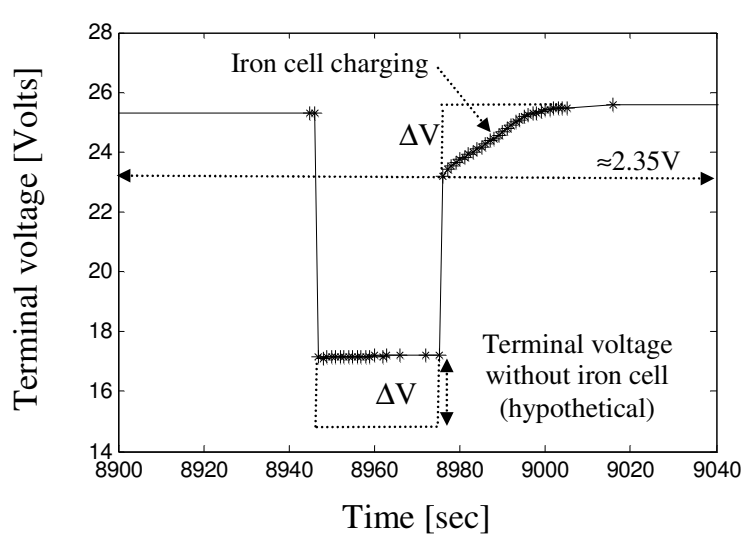

Fig. 11. Enlarged high power transient terminal voltage pulse

The iron cell resistance can be estimated as a function of DOD_Fe by subjecting the module to a high power pulse discharge sequence of $60 \mathrm{~min}$ at $2.5 \mathrm{~A}, 30 \mathrm{sec}$ at maximum current followed by $30 \mathrm{~min}$ open circuit. The voltage progression is shown in Fig. 10, where the minimum terminal voltage is governed by the BMI and set at 0.66 nominal OCV $(17.2 \mathrm{~V})$. One of the voltage transients during a maximum current pulse shown in Fig
10 (marked) is enlarged in Fig. 11, alongside a method for calculating the iron cell resistance during charging and discharging events. It should be noted that based on this method, the accuracy of the estimate iron cell resistance is entirely based on the accuracy of the previously estimated nickel resistances.

Figure 12 shows the estimated resistive circuit parameters as a function of layer radius during discharging at $295^{\circ} \mathrm{C}$. It can be seen that the nickel cell resistances appear to sharply fall, whilst the iron cell resistance rises when $\mathrm{Sr}<0.3$. The anomaly is due to the difficulty in determining the nickel cell parameters with increased DOD. Although this does not affect the accuracy of the model, it does not represent the likely progression of actual resistances, which has been added for clarity. Additionally from Fig. 12, it can be seen that when the layer radius is large and the +ve electrode exhibits minimum resistance, the current contribution of the iron cell is small, since its equivalent resistance is high. As the layer radius reduces the iron resistance reduces significantly. This has the effect of increasing the current contribution of the iron cell and counteracting the effects of the higher nickel cell resistances as the layer radius reduces (i.e. the cell maintains good pulse power capability at high DOD levels). For completeness, Fig. 13 shows an example of the estimated OCV as a function of DOD.

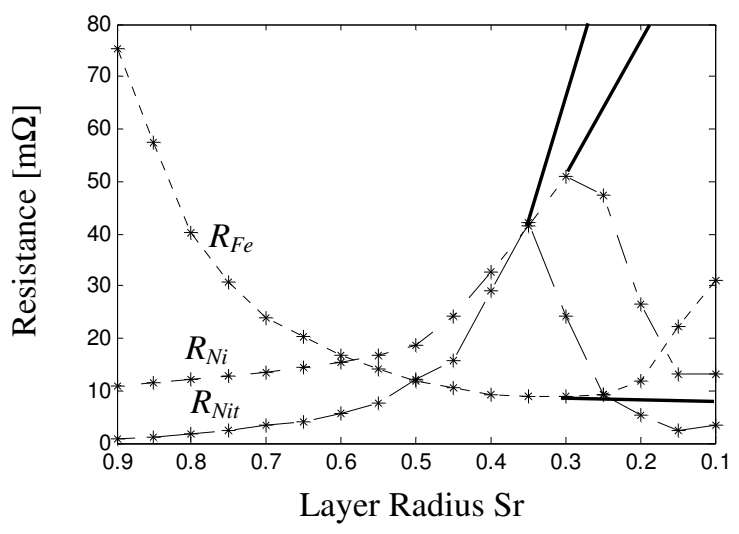

Fig. 12. Equivalent circuit parameters of the doped Zebra cell at $295^{\circ} \mathrm{C}$

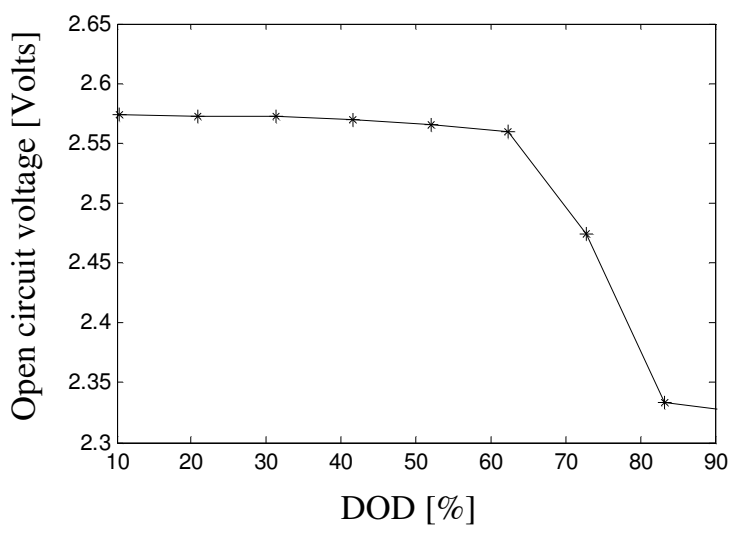

Fig. 13. Estimated open circuit voltage of the doped Zebra cell at $295^{\circ} \mathrm{C}$ 


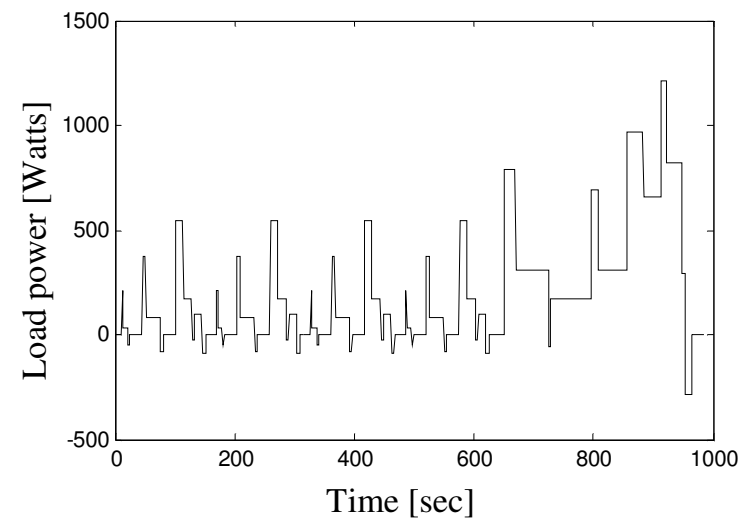

Fig. 14. Estimated and scaled smart car power requirements during a NEDC driving cycle

In order to evaluate the performance of the Zebra battery model an ECE+EDDC (NEDC) driving cycle is employed as a benchmark. Using data from the smart car drive train, a demand power profile was estimated over one NEDC cycle and scaled, Fig 14, to suit the 10 cell experimental Zebra module. Figures 15 and 16 show both the simulated and experimental results for the terminal voltage and terminal current progression throughout the cycle.

\section{CONCLUSIONS}

This paper describes the operational behaviour and advantages of the high temperature, Zebra battery, for use in all electric urban vehicles. It is shown that an equivalent parallel electrical circuit can be employed to accurately simulate the electrochemical behaviour inherent in the most recent generation of Zebra cells. The experimental procedure is outlined and summary attributes of the investigation validated by both simulation studies, and experimentally, via measurements from a prototype battery module intended for use in a all electric smart car and benchmark NEDC urban driving cycle. It is demonstrated that the transient behaviour during charging and discharging can be predicted with sufficient accuracy to optimise the smart car drive train without time consuming experimental trials.

\section{REFERENCES}

[1] N. Amamm, J. Bocker and F. Prenner, "Active damping of drive train oscillations for an electrically driven vehicle," IEEE/ASME Trans. on Mechatronics, Vol. 9, No. 4, pp. 697-700 (2004).

[2] C-H. Dustmann "Advances in ZEBRA batteries," Journal of Power Sources, Vol. 127, pp 85-92, (2004).

[3] J.L. Sudworth "The sodium/nickel choride (ZEBRA) battery," Journal of Power Sources, Vol. 100, pp 149-163, (2001).

[4] P. Lailler, J-F Sarrau, C. Sarrazin "Comparative study for "36v" vehicle applications: Advantages of lead-acid batteries," Journal of Power Sources, Vol. 95 pp. 58-67, (2001).

[5] M. Wansner, "Energy production industry and power station engineering”, Institute for Power Engineering, Munich Technical University, Thesis, (1996).

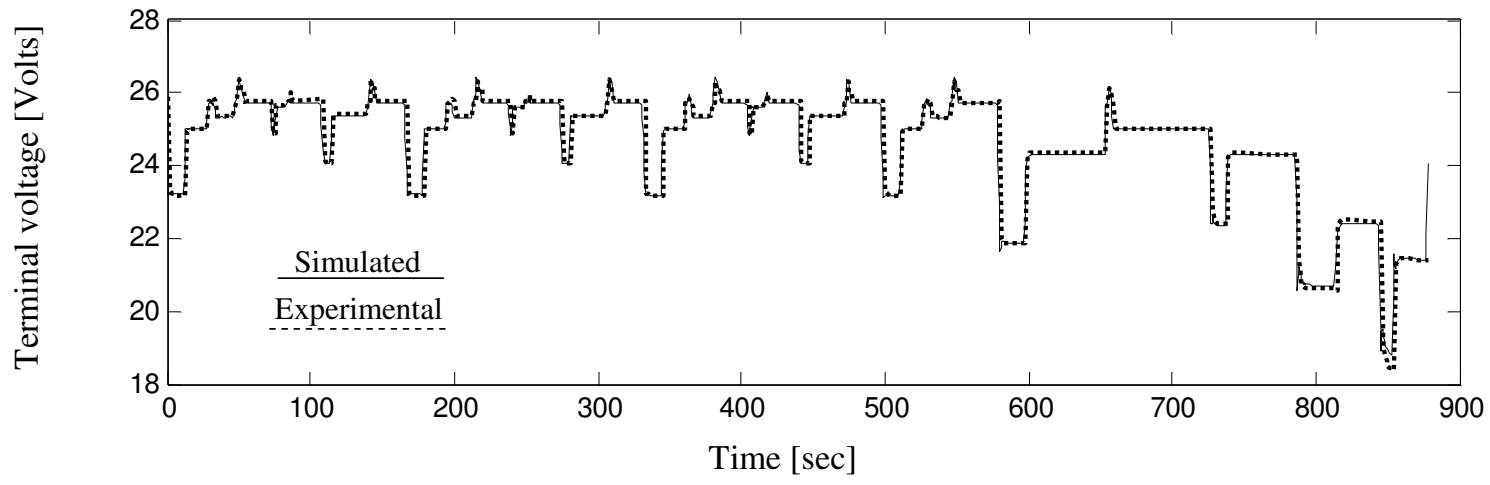

Fig. 15. Simulated and experimental terminal voltage of a 10 cell Zebra module during a NEDC driving cycle

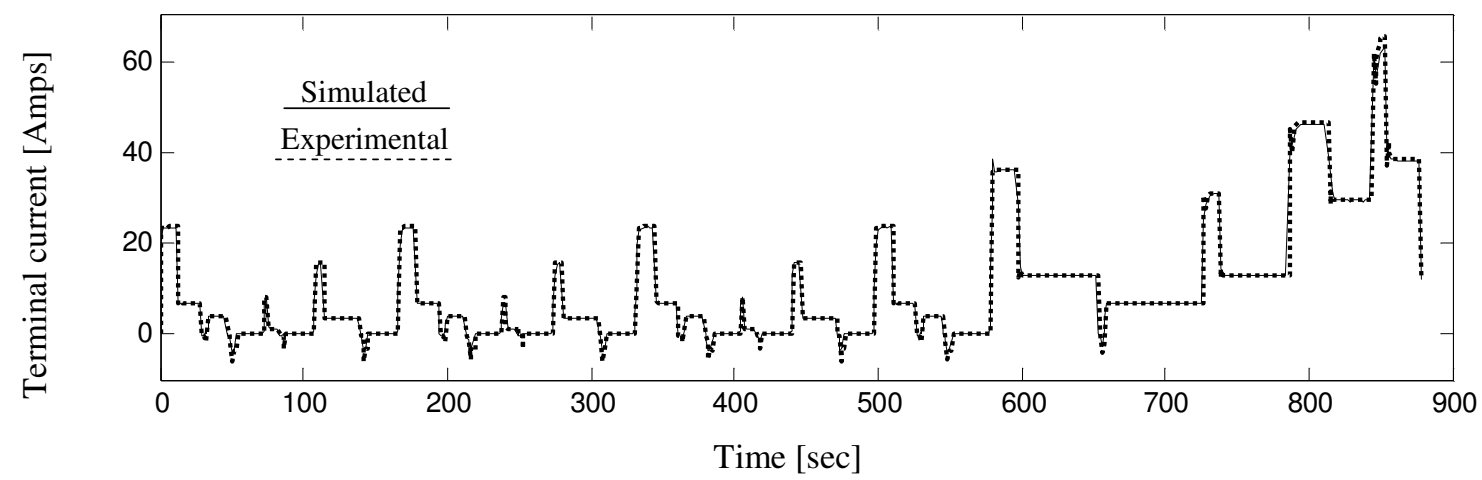

Fig. 16. Simulated and experimental terminal current of a 10 cell Zebra module during a NEDC driving cycle 